\title{
Chromosome aberrations in coeliac and non-coeliac enteropathies
}

\author{
S Kolacek, I Petkovic, I W Booth
}

\begin{abstract}
The frequency of chromosomal aberrations in peripheral blood lymphocytes was assessed in three groups of children: untreated coeliac disease $(n=20)$; noncoeliac disease enteropathies $(n=15)$; controls $(n=15)$. The mean frequency of aberrant cells and the total number of aberrations per 100 metaphases was increased in the coeliac disease group compared with controls by factors of 5 and 6 , respectively ( $p<0.01$ for both). Aberrant cells and total aberrations were similarly increased in the non-coeliac disease enteropathy group by a factor of 3.7 in each case $(p<0.05)$. However, the frequency of aberrations in the two enteropathy groups was not significantly different. Children with coeliac disease, similar to affected adults, have evidence of increased chromosomal instability. However, similarly increased chromosomal aberrations are seen in children with non-coeliac disease enteropathies, indicating that the abnormality is not specific for coeliac disease. (Arch Dis Child 1998;78:466-468)
\end{abstract}

Keywords: coeliac disease; chronic enteropathy; chromosomal aberrations

Adults with coeliac disease have an increased incidence of malignancy: compared with the general population, they have a 43-fold increased risk of non-Hodgkin's small bowel lymphoma, a 12-fold increase in the risk of oesophageal carcinoma, and a 10-fold increased risk of developing cancer of the oropharynx. ${ }^{1}$ Overall, $8-13 \%$ of patients with coeliac disease develop a malignancy. ${ }^{2}$ It has been suggested that coeliac disease should be regarded as a premalignant condition ${ }^{3}$; however, the risk of malignancy is greatly diminished by a gluten free diet-patients adhering to a strict gluten free diet for at least five years have a similar risk to the general population. ${ }^{4}$

The mechanisms of this increased cancer risk in coeliac disease patients remains speculative. In the only cytogenetic study in coeliac disease, Fundia et al found an increased number of chromosomal aberrations compared with healthy controls. ${ }^{5}$ Moreover, chromosomal instability tended to occur at specific cancer breakpoints, suggesting that this fragility could be related to the predisposition to cancer in patients with coeliac disease. ${ }^{6}$ Similar studies in children have not been performed but may be particularly illuminating. First, children have not been exposed to the effect on genomic instability of factors such as aging and environmental carcinogens. Second, infants and children have a higher incidence of transient, non-coeliac enteropathies, thus providing an additional and potentially valuable control group.

We assessed the frequency of chromosomal aberrations in peripheral blood lymphocytes of children with untreated coeliac disease and compared it with the frequency in children with non-coeliac disease enteropathies and controls.

\section{Methods}

Cytogenetic analyses were performed in three groups: children with coeliac disease; children with other non-coeliac disease enteropathies; children without organic disease (table 1). Exclusion criteria were intercurrent viral illness and antibiotic treatment.

CHILDREN WITH COELIAC DISEASE

We studied prospectively 20 consecutive patients with newly diagnosed and untreated coeliac disease. Diagnostic criteria were a typical abnormality on small bowel biopsy (hyperplastic villous atrophy with increased intraepithelial lymphocytes), positive antigliadin and antiendomysial antibodies (Eurospital, Italy), and improvement (clinical and anthropometric) on a gluten free diet.

CHILDREN WITH NON-COELIAC ENTEROPATHIES This group comprised 15 patients admitted to hospital for investigation of chronic diarrhoea and weight loss. The final diagnoses, and diagnostic criteria for each were as follows.

\section{Postenteritis syndrome}

There were nine children in this group, mean (SD) age 32 (19) months. They had acute onset diarrhoea, leading to protracted diarrhoea of more than one month duration; abnormal small intestinal biopsy (abnormal villous architecture, with mucosal inflammation, but not subtotal villous atrophy); absent antiendomysial antibodies; and recovery on a cow's milk free, gluten containing diet.

\section{Cow's milk protein intolerance}

There were three children in this group, mean age 2.3 (1.4) months. They had protracted diarrhoea and failure to thrive following the introduction of cow's milk; no history of previous exposure to dietary gluten; resolution of diarrhoea and start of catch up growth within seven days of introducing a cow's milk free diet. Small intestinal biopsies were not performed in this group as biopsy was not part of the routine 
Table 1 Characteristics of subjects and controls

\begin{tabular}{llllll}
\hline & No & $\begin{array}{l}\text { Sex } \\
(M / F)\end{array}$ & $\begin{array}{l}\text { Mean age } \\
\text { (months) }\end{array}$ & Age range & $\begin{array}{l}\text { Mean } \mathrm{W} / \mathrm{Hz} \\
\text { score }(S D)\end{array}$ \\
\hline A. Coeliac disease & 20 & $8 / 12$ & 23 & $7 \mathrm{~m}-6 \mathrm{y}$ & $-2.09(0.81)$ \\
B. Non-coeliac enteropathy & 15 & $9 / 6$ & 24 & $1 \mathrm{~m}-7.5 \mathrm{y}$ & $-1.76(0.73)$ \\
C. Controls & 15 & $9 / 6$ & 51 & $5 \mathrm{~m}-14 \mathrm{y}$ & $-0.31(1.41)$ \\
\hline
\end{tabular}

W/H (weight for height): A $v$ B: $\mathrm{p}>0.05$; A $v$ C: $\mathrm{p}<0.01 ; \mathrm{B} v \mathrm{C}: \mathrm{p}<0.01$.

clinical management of this disorder and so is not ethically justified. The enteropathy has been well described.?

Idiopathic protracted diarrhoea

There were three children with this diagnosis, mean age 3.5 (1.2) months. They had protracted diarrhoea and severe failure to thrive; abnormal small intestinal biopsy (disturbed villous architecture, mucosal inflammation but not subtotal villous atrophy); absent antiendomysial antibodies; failure to resolve on a cow's milk free diet; subsequent improvement on a semi-elemental diet, with continuing resolution of symptoms following the introduction of dietary gluten.

CONTROL GROUP

Fifteen children undergoing investigation for various symptoms (including anorexia, short stature, recurrent abdominal pain, constipation, and arthralgia), who were subsequently not shown to have organic disease. Small intestinal biopsies $(n=3)$ and antiendomysial antibody analyses $(n=3)$ were performed when clinically indicated and were all normal.

\section{PROCEDURES}

Chromosomal aberrations were analysed in peripheral blood lymphocytes. Cells were grown in Eagle's minimal essential medium supplemented with $20 \%$ human AB serum and phytohaemagglutinin and incubated for 72 hours at $37^{\circ} \mathrm{C}$. Chromosome preparations were obtained by a standard method including colchicine treatment, hypotonic treatment with $0.075 \mathrm{M} \mathrm{KCl}$, followed by fixation with methanol:acetic acid (3:1). Air dried slides were stained by the GTG banding method. For each subject, 100 metaphases were analysed for chromosome aberrations (breaks, gaps, and acentric fragment). The analyses were performed by a single cytogeneticist who was blinded to the origin of the cells and was not involved in the treatment of the patients.

As the variables measured were not normally distributed, the significance of the differences between groups in the frequency of aberrant cells and the total number of aberrations was determined with the non-parametric median test. Intergroup differences in nutritional status were expressed as weight for height $\mathrm{z}$ scores, and tested with the Student's $t$ test.

Table 2 Mean (SD) percentage of aberrant cells, and number of aberrations per 100 metaphases

\begin{tabular}{lll}
\hline & Aberrant cells (\%) & $\begin{array}{l}\text { Aberrations/100 } \\
\text { metaphases }\end{array}$ \\
\hline A. Coeliac disease & $1.35(1.38)$ & $1.55(1.5)$ \\
B. Non-coeliac enteropathy & $1.00(1.06)$ & $1.00(1.05)$ \\
C. Controls & $0.27(0.45)$ & $0.27(0.45)$ \\
\hline
\end{tabular}

Intergroup differences: A $v \mathrm{C}, \mathrm{p}<0.01 ; \mathrm{A} v \mathrm{~B}, \mathrm{p}>0.05 ; \mathrm{B} v \mathrm{C}, \mathrm{p}<0.05$.

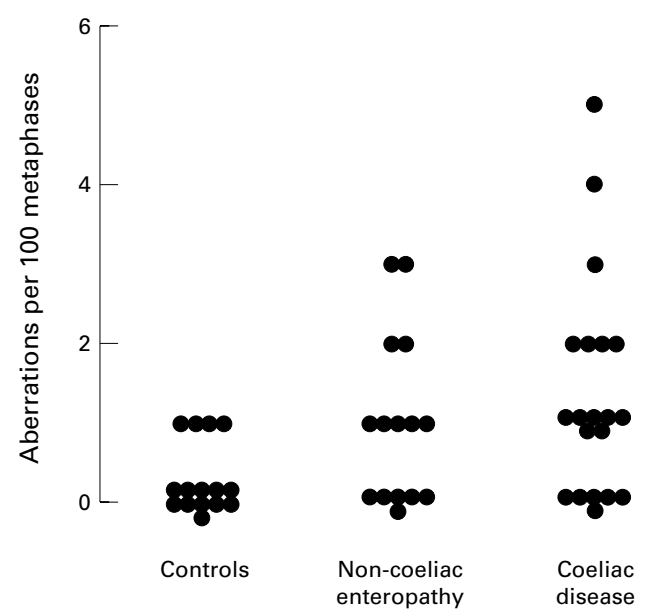

Figure 1 Distribution of individual values of aberrations per 100 metaphases in each group.

\section{Results}

Table 2 shows the mean percentage of aberrant cells and aberrations per 100 metaphases for the three groups. The mean frequency of aberrant cells and the total numbers of aberrations per 100 metaphases were higher in the coeliac disease group compared with controls by factors of 5 and 6 , respectively $(p<0.01$ for both). Aberrant cells and total aberrations were similarly increased in the non-coeliac disease enteropathy group by a factor of 3.7 in both cases $(p<0.05)$. However, the frequency of aberrations in the two enteropathy groups was not significantly different.

In both enteropathy groups most of the patients had at least one aberrant cell (coeliac disease group $70 \%$; non-coeliac disease group $60 \%$ ), while in the control group only $26 \%$ had aberrant cells (fig 1). In the coeliac disease group there were 27 breaks and four gaps. Only breaks were detected in the non-coeliac disease enteropathy group and in controls (15 breaks in the non-coeliac disease enteropathy group, four in the control group).

From the mean weight for height $\mathrm{z}$ scores, both enteropathic groups were significantly wasted compared with controls $(p<0.01$ in each case) (table 1).

\section{Discussion}

Our study confirms and extends the original observation by Fundia et al that adults with coeliac disease have chromosomal aberrations. ${ }^{56}$ It is now clear that affected children with untreated coeliac disease also show increased chromosomal instability. Moreover, our data indicate for the first time that children with non-coeliac enteropathies have similar abnormalities in peripheral blood lymphocytes.

Fundia et al showed that $24 \%$ of cells from a group of 12 adults (mean age 30 years) with untreated coeliac disease had evidence of chromosomal aberrations, compared with $4 \%$ of cells from a group of eight control subjects (mean age 34 years). ${ }^{6}$ The incidence of abnormality in coeliac disease was substantially higher than in our study, which may reflect an effect of aging on the frequency of chromosomal aberrations. Moreover, the mucosal 
lesions in their adult patients may have been present for considerably longer before the diagnosis was made-most of the patients in our study had a history of only a few months. We note, however, that the ratio of chromosomal abnormality between coeliac disease and controls was identical in the two studies. Fundia $e t$ al also found that the aberrations in their study clustered significantly at known cancer break points. ${ }^{6}$ Unfortunately, the much lower frequency of aberrations in the children we studied prevented us from analysing that aspect.

By studying infants and children with coeliac disease we were able to compare our results with an age compatible group with a heterogeneous mixture of non-coeliac enteropathies. The observation that fragility was similarly increased in this non-coeliac disease group suggests that the chromosomal lesion is non-specifically associated with enteropathy and is unlikely to be a primary (that is, genetically) determined abnormality in coeliac disease. It has been suggested that the chromosomal instability seen in coeliac disease may be related to the well recognised increase in malignancy in this disorder. ${ }^{5}$ Our observation that genomic instability in coeliac disease is probably secondary (not primary) to the enteropathy is consistent with studies indicating that the increased risk of cancer reverts to normal after more than five years on a strict gluten free diet. ${ }^{4}$ In contrast to coeliac disease, in which the enteropathy in the presence of dietary gluten is life long, the mucosal lesions in our noncoeliac disease enteropathy group were almost certainly transient, which may explain the absence of reports of increased malignancy in this group of enteropathies. A report of an increased risk of cancer in relatives of patients with coeliac disease ${ }^{8}$ seems to be more consistent with an explanation based on a common, genetically determined predisposition. However, an alternative explanation is that at least some of the relatives with malignancy had subclinical coeliac disease.

There are various limitations to our study. First, nutritional status in the two enteropathy groups was significantly worse than in the controls. While this is a potentially confounding variable that should be addressed in future studies, we have not been able to find any evidence that wasting increases genomic instability on its own; nor does it invalidate our central observation that genomic instability is not specific to coeliac disease. Second, the diagnosis in our coeliac disease patients younger than 2 years did not comply strictly with the European Society of Paediatric Gastroenterology and Nutrition diagnostic criteria. ${ }^{9}$ However, not only did all patients have the typical mucosal appearances and clinical response to gluten withdrawal, but they also all had antiendomysial antibodies - a serological marker with a specificity for coeliac disease approaching $100 \% .^{10}$ Therefore, we think it is implausible that these patients did not have coeliac disease. Finally, our controls were significantly older than our subjects. However, had there been an age related effect in this group, it would have reduced rather than enhanced the differences we observed.

The basis for and implications of our observations are uncertain. We have no direct evidence that the increased genomic instability in coeliac disease is responsible for the observed increased rate of malignancy. While an increase in mitotic rate of lymphocytes (and enterocytes) in the small intestinal mucosa may be one factor associated with malignant transformation leading to small bowel lymphoma (and carcinoma), ${ }^{11}$ it does not explain the increase in foregut malignancies seen in coeliac disease.

We recommend that further work should include studies both on peripheral blood and mucosal lymphocytes from patients with coeliac disease undergoing gluten challenge, and in non-coeliac disease relatives of patients with coeliac disease. Work aimed at determining the specificity of this genomic abnormality with respect to mucosal inflammation could include studies of peripheral blood lymphocytes from patients with inflammatory bowel disease, in whom long standing mucosal inflammation is associated with an increased risk of malignancy.

1 Holmes GKT, Stokes PL, Sorahan TM, et al. Coeliac disease, gluten free diet and malignancy Gut 1976;17:61219.

2 O'Driscoll BRC, Stevens FM, O'Gorman TA, et al. HLA type of patients with coeliac disease and malignancy in the west of Ireland. Gut 1982;23:662-5.

3 Marsh MN. Is coeliac disease (gluten sensitivity) a premalignant condition? $f$ Pediatr Gastroenterol Nutr premalignant $c$ c

4 Holmes GKT, Prior P, Lane MR, et al. Malignancy in coeliac disease - effect of a gluten free diet. Gut 1989;30:3338.

5 Fundia A, Gonzales CMB, Bai JC, et al. Chromosome instability in lymphocytes from patients with celiac disease. Clin Genet 1994;45:57-61.

6 Fundia A, Gomez JC, Maurino E, et al. Chromosome instability in untreated adult coeliac disease patients. Acta Paediatr Suppl 1996;412:82-4

7 Walker-Smith JA. Food-related disorders. In: Walker-Smith JA, Hamilton WA, eds. Practical pediatric gastroenterology. Hamilton: BC Decker, 1996:180-9.

8 Stokes PL, Prior P, Sorahan TM, McWalter RJ, Waterhouse JAH, Cooke WT. Malignancy in relatives of patients with coeliac disease. Br f Prev Soc Med 1976;30:17-21.

9 Working Group of the European Society of Paediatric GasWorking Group of the European Society of Paediatric Gas-
troenterology and Nutrition. Revised criteria for diagnosis troenterology and Nutrition. Revised criteria for diag disease. Arch Dis Child 1990;65:909-11.

10 Grodzinsky E. Serological markers in subclinical and clinical gluten enteropathy. Linkoping: Linkoping University Medical Dissertations No 422 Edsbruk, Sweden, 1994.

11 Holmes GKT. Celiac disease and malignancy. $\mathcal{F}$ Pediatr Gastroenterol Nutr 1997;24:S20-4. 